procurer et peuvent être mis entre les mains de manipulateurs non spécialisés.

Il est à souhaiter que cette méthode trouve auprès des organismes de contrôle chargés de dépister l'addition de laits étrangers au lait humain tout l'appui qu'ils désirent, toute la commodité et la rapidité d'exécution qu'ils souhaitent.

\title{
LA MÉTHODE DE CONTROLE ET DE CONSERVATION \\ DU LAIT MATERNEL AU LACTARIUM (1)
}

par

A. ROSSIER

Médeoin des Hôpitaux de Paris

\section{JACQUELINE BERTRAND}

Directrice technique du Lactarium

Le Lactarium de l'Ecole de Puériculture compte aujourd'hui deux années d'existence (2)

Fondé en avril 1947 pour répondre à une demande pressante, il peut actuellement, grâce à une organisation de plus en plus poussée, assurer du lait maternel aux nourrissons débiles, prématurés et malades qui en ont un besoin vital.

Le principe même du lactarium, qui est de collecter le lait maternel à domicile, suppose et exige la mise en œuvre, d'une manière systématique, d'une série d'examen's de contrôle pratiqués chaque jour au laboratoire spécialisé du lactarium.

Nous voulons, dans cet article, exposer la méthode de contrôle et de conservation du lait maternel.

\section{CONTROLE DE LA PROPRETÉ}

\section{A. Acidimétrie}

Un test indirect, mais très sensible de la propreté du lait récolté est fourni par son taux d'acidité. Il renseigne, en effet, indirectement sur la mánière dont le lait a été recueilli. Une hygiène défectueuse de la traite, une conservation dans un récipient insuffisamment nettoyé ou à une température insuffisamment fraîche se traduira, en effet, par un excès d'acidité dû au développement de la flore lactiquel de fermentation. Il peut même arriver, dans les conditions les moins bonnes, que le lait “ tourne ». L'acidité est, d'autre part, plus élevée en été qu'en hiver.

Normalement, un bon lait maternel doit renfermer moins de

(1) L'Alimentation et la Vie, 1949, 37, 4-5-6-111.

(2) Voir Le Lait, 1948, 28, 333. 
0 gr. 4 d'acide lactique par litre, ou encore doit titrer moins de $4^{\circ}$ Dornic.

La méthode rapide, simple, instantanée permettant un dégrossissage suffisant est la méthode au tournesol, appliquée chaque jour t̀ tous les échantillons de lait.

Une baguette de verre stérile est trempée dans chaque biberon et la goutte qu'elle ramène est posée sur une feuille de papier de tournesol bleu, recouverte, pour plus de facilité, d'une grille métallique percée de trous d'environ $15 \mathrm{~mm}$. de diamètre. Si la tache reste bleutée, on admet que le lait a un degré Dornic inférieur à 4. Si la tache devient rouge ou rose, on parfait cette approximation par un dosage.

La méthode précise, consiste à doser l'acidité par la soude. $10 \mathrm{~cm}^{3}$ de lait additionné de quelques gouttes de phénol phtaléine sont neutralisés jusqu'à coloration rose persistante par $\mathbf{n} \mathbf{c m}^{3}$ de soude Dornic (soude $\mathrm{N} / 9$ ). Cette soude est titrée de telle façon que $1 / 10^{\mathrm{e}}$ de centimètre cube de soude Dornic, équivaut à $1^{\circ}$ Dornic, soit à 0 gr. 10 d'acide lactique par litre.

Au-dessus de $12^{\circ}$ Dornic, soit $1 \mathrm{gr}$. 20 d'acide lactique, on admet que le lait est impropre à être consommé. Entre 4 et $12^{\circ}$, le lait est consommable, mais il sera conservé le moins longtemps possible. Après stérilisation à $100^{\circ}$ il sera distribué dans le premier lot. Audessous de $4^{\circ}$, lait excellent qui pourra être mis en stock.

Notre expérience nous a donné, en moyenne, $80 \%$ de laits inférieurs à $4^{\circ}$ Dornic, $20 \%$ seulement supérieurs à $4^{\circ}$ Dornic.

Les laits titrant au-dessus de $12^{\circ}$ (laits impropres) ne sont pas conservés et sont jetés. Ils représentent, en moyenne, $6 \%$ de la collecte quotidienne et peuvent atteindre, aux jours très chauds, de 7 à $8 \%$.

\section{B. Test bactériologique}

Un second test de propreté est la numération des germes se trouvant dans le lait frais apporté au Lactarium. On peut le faire par ensemencement du lait à dilutions croissantes sur boîtês de Pétri.

Une méthode plus simple (procédé de Burri) consiste à employer une anse de platine jaugée, contenant $1 \mathrm{mgr}$, de lait qu'on ensemence en stries sur gélose inclinée.

Après 48 heures d'étuve à $37^{\circ}$, on lit le nombre des colonies et on multiplie par 1.000 pour avoir le nombre de germes vivants par centimètre cube.

Ce contrôle est établi tous les quinze jours pour chaque donneuse et donne une idée approchée, pour chacune, de la propreté de la récolte. 
On considère comme prélevé proprement, un lait contenant moins de 10.000 germes par centimètre cube.

Si l'on trouve plus de 100.000 germes, la donneuse est l'objet d'une surveillance et d'une visite de l'Assistante Sociale.

Un lait contenant 1.000 .000 de germes au centimètre cube est rejeté ; une enquête est faite et si- les résultats sont défavorables, la donneuse est rayée du Lactarium.

Les germes ordinairement trouvés sont presque toujours des microbes Gram + du type B. lactiques.

En moyenne, les numérations nous ont donné dans la majorité des cas, moins de 50.000 germes au centimètre cube, et, en été, moins de 100.000 .

\section{CONTROLE DE LA PURETÉ ; DÉTECTION DU MOUILLAGE}

\section{A. Densimétrie}

Méthode simple, rapide, qui nécessite seulement la lecture du densimètre plongé dans l'éprouvette remplie de lait, mais exige, évidemment, une manipulation pour chaque biberon. Aussi n'estelle pas pratiquée tous les jours sur tous les biberons, mais faite pour chaque donneuse tous les 4 jours, soit huit fois par mois. Normalement, le lait de femme a une densité supérieure à 1.030. Au-dessous de 1.028 , il y a présomption de mouillage. Nous trouvons habituellement des chiffres étagés entre 1.029 et 1.033 . Ils sont à peu près invariables chez la même nourrice.

\section{B. Dosage du taux butyreyx et de l'extrait sec}

Un lait ayant une densité inférieure à 1.030 n'est pas foreément un lait mouillé : il peut être un lait anormalement riche en graisses. Taux butyreux.

On dissout la caséine de $11 \mathrm{~cm}^{3}$ de lait maternel dans $10 \mathrm{~cm}^{3} \mathrm{de}$ $\mathrm{SO}^{4} \mathrm{H}^{2}$ de densité 1.820 à 1.825 . On ajoute $1 \mathrm{~cm}^{3}$ d'alcool amylique purifié pour favoriser la séparation du beurre. On mesure ensuite dans le tube gradué spécial la matière grasse après centrifugation 3 minutes.

On lit au bain-marie à $65^{\circ}$

Les chiffres obtenus sont très variables, et l'on sait que rien n'est plus changeant, même chez une même nourrice, que le taux des graisses du lait qui peut aller de 20 à $60 \mathrm{gr}$, par litre. Aussi bien la vérification absolue du mouillage ne peut-elle se faire que grâce à une donnée supplémentaire : le résidu sec.

Résidu sec dégraissé.

On le calcule à l'aide d'une règle à calculer spéciale, la règle de Gerber, en fonction de la densité et du taux butyreux. Le chiffre 
obtenu doit être supérieur ou égal à 8, pour permettre d'éliminer la fraude par addition d'eau.

On conclut à la fraude s'il est inférieur à 7,6.

Entre 7,6 et 8 , il y a suspicion et une enquête est faite, dont le but essentiel est la récolte du lait suspect sous contrôle direct de la visiteuse et l'analyse du lait ainsi recueilli.

Nous avons décelé une fraude par mouillage depuis le début du fonctionnement du Lactarium.

\section{CONTROLE DE LA FRAUDE PAR ADDITION DE LAIT DE VACHE}

\section{A. Test rapide aux U.-V.}

Ce test est pratiqué systématiquement chaque jour pour tous les échantillons de lait. Il est basé sur la fluorescence bleue violette du lait de femme, jaune canari du lait de vache aux radiations U.-V. Le lait de vache contient, en effet, beaucoup plus de vitamine B2 que le premier, et c'est la lactoflavine qui est responsable de cette intense couleur jaune. Nous utilisons une lampe à rayons U.-V. munie d'un écran de Wood en verre de Hanau, perméable aux radiations d'ondes inférieures à 366 millimicrons. La différence de coloration est, en général, évidente. Elle est encore nette lorsque le lait de femme contient $10 \%$ de lait de vache.

Elle est parfois douteuse : dans $5 \%$ des cas.

Ces cas douteux néeessitent alors une épreuve supplémentaire plus précise.

\section{B. Test biologique}

C'est une épreuve de précipitation par un sérum de lapin spécialement préparé par des injections de lait de vache. Le sérum acquiert un pouvoir précipitant vis-à-vis de ce lait (JORDANOFF et KoschUCHANOFF, 1932).

On injecte à un lapin, 5 jours de suite, tous les jours, $5 . \mathrm{cm}^{3}$ de lait de vache écrémé stérile dans la veine marginale.

5 jours après la $5^{\text {e }}$ injection, on saigne le lapin, on récolte le sérum et on le met en petites ampoules d' $1 \mathrm{~cm}^{3}$, après addition de $0,5 \%$ de phénol. On conserve à la glacière et à l'obscurité.

Pour faire le test, on mélange sur une lame une goutte du lait suspect écrémé et 4 gouttes du sérum de lapin. Une agglutination d'une netteté absolue se produit en quelques instants lorsque le lait contient du lait de vache. La méthode est sensible pour une proportion de $5 \%$ de lait animal.

Quand la fraude est suspectée, une surveillance rigoureuse est exercée pendant plusieurs jours (tout-en rejetant le lait suspect) et une épreuve biologique est faite dans une tétée prélevée sous contrôle. La donneuse est renvoyée. 
Nous avons déecelé, jusqu'à présent 4 fraudes de ce genre.

\section{BOUCHAGE}

Aprés ces divers contrôles, chaque biberon est bouché par une capsule d'aluminium sertie automatiquement grâce à un appareil spécial : ce bouchage hermétique permet l'immersion complète du biberon dans le stérilisateur.

Un bouchon en caoutchouc épais conçu par le $\mathrm{D}^{\mathrm{r}}$ LEgros de Liége, permet de faire le vide dans le biberon et d'effectuer une stérilisation sous vide encore plus parfaite.

\section{STÉRILISATION}

Les biberons obturés sont alors stérilisés.

En règle, tous les laits dont le degré Dornic est inférieur à $4^{\circ}$ subissent la Tyndallisation, à $65^{\circ}$, pendant 20 minutes, répétée 3 jours de suite, suivie chaque fois d'un refroidissement brusque.

La stérilisation à $100^{\circ}$ est réservée aux laits titrant entre $4^{\circ}$ et $12^{\circ}$ Dornic.

\section{VI, CONSERVATION}

Le lait de consommation courante est conservé en armoire frigorifique à une température de 0 à $+4^{\circ}$.

Le lait destíné à être stocké pour être disponible aux pointes de consommation durant les périodeș de grande chaleur est congelé à - $30^{\circ}$ pendant 35 minutes dans un congélateur spécial à alcool. Grâce à ce procédé de congélation rapide il ne subit aucune altération et retrouve son homogénéité normale après décongélation lente au bain-marie.

\section{RÉSULTATS}

A l'heure actuelle (mars 1949), le nombre de donneuses de lait s'élève à 160 . La collecte mensuelle s'élève à 1.421 litres (45,8 litres, par jour. La vente mensuelle atteint 1.337 litres.

Le stock permanent est de 105 litres, 169 enfants bénéficient du lait du Lactarium.

Les résultats obtenus avec le lait de Lactariūm, en particulier chez les grands prématurés débiles, montrent que ce lait ñ'a rien perdu de sés qualités spécifiques puisqu'il assure un état de nutrition parfait et une croissance pleinement satisfaisante.

(Travail de la chaire de Clinique de Puériculture.

Professeur : Marcel Lelong.) 